\title{
BMJ Open Emergency obstetric care provision in Southern Ethiopia: a facility-based
}

\section{survey}

To cite: Windsma $M$, Vermeiden T, Braat F, et al. Emergency obstetric care provision in Southern Ethiopia: a facility-based

survey. BMJ Open

2017;7:e018459. doi:10.1136/

bmjopen-2017-018459

- Prepublication history for this paper is available online. To view please visit the journal online (http://dx.doi.org/10. 1136/bmjopen-2017-018459).

Received 30 June 2017 Revised 8 October 2017 Accepted 13 October 2017

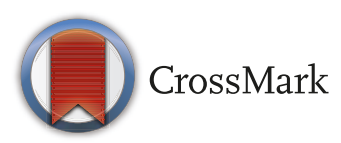

${ }^{1}$ Faculty of Medical Sciences, Universitair Medisch Centrum Groningen, University of Groningen, Groningen, The Netherlands

${ }^{2}$ Maternity Department, Butajira General Hospital, Butajira,

Ethiopia

${ }^{3}$ Department of Health Sciences, Global Health, University Medical Centre Groningen, University of Groningen, Groningen, The Netherlands

${ }^{4}$ Ethiopia Country Office, UNICEF, Addis Ababa, Ethiopia

${ }^{5}$ Department of Obstetrics,

Leiden University Medical

Centre, Leeuwarden, The

Netherlands

${ }^{6}$ Department of Obstetrics and

Gynaecology, Leeuwarden

Medical Centre, Leeuwarden,

The Netherlands

Correspondence to

Tienke Vermeiden;

c.j.vermeiden@umcg.nl

\section{ABSTRACT}

Objectives To assess the provision of basic emergency obstetric and newborn care (BEmONC), knowledge of high-risk pregnancies and referral capacity at health centres in Southern Ethiopia.

Design A facility-based survey, using an abbreviated version of the Averting Maternal Death and Disability needs assessment tool for emergency obstetric and newborn care. Modules included infrastructure, staffing, number of deliveries, maternal and perinatal mortality, BEmONC signal functions, referral capacity and knowledge of risk factors in pregnancy.

Setting Primary healthcare centres providing delivery services in the Eastern Gurage Zone, a predominantly rural area in Southern Ethiopia.

Participants All 20 health centres in the study area were selected for the assessment. One was excluded, as no delivery services had been provided in the 12 months prior to the study.

Results Three out of 19 health centres met the government's staffing norm. In the 12 months prior to the survey, $10004(\bar{X}=527 \pm 301)$ deliveries were attended to at the health centres, but none had provided all seven BEmONC signal functions in the three months prior to the survey $(\overline{\mathrm{X}}=3.7 \pm 1.2)$. Eight maternal and 32 perinatal deaths occurred. Most health centres had performed administration of parenteral uterotonics (17/89.5\%), manual removal of placenta (17/89.5\%) and neonatal resuscitation (17/89.5\%), while few had performed assisted vaginal delivery $(3 / 15.8 \%)$ or administration of parenteral anticonvulsants (1/5.3\%). Reasons mentioned for non-performance were lack of patients with appropriate indications, lack of training and supply problems. Health workers mentioned on average $3.9 \pm 1.4$ of 11 risk factors for adverse pregnancy outcomes. Five ambulances were available in the zone.

Conclusion BEmONC provision is not guaranteed to women giving birth in health centres in Southern Ethiopia. Since the government aims to increase facility deliveries, investments in capacity at health centres are urgently needed.

\section{BACKGROUND}

In 2015, 303000 maternal deaths, 2.6 million stillbirths and 2.7 million early neonatal deaths were estimated to have occurred

\section{Strengths and limitations of this study}

- First basic emergency obstetric and newborn care (BEmONC) assessment in Ethiopia since 2008, using a validated Averting Maternal Death and Disability tool.

- Wide range of modules assessed: infrastructure, staffing, number of deliveries, maternal and perinatal mortality, BEmONC signal functions, referral capacity and knowledge of risk factors in pregnancy.

- Survey included all 20 health centres in Eastern Gurage Zone, Ethiopia.

- The number of health centres and area of study were relatively small.

- Methods included interviews with health centre staff, which are vulnerable to recall bias.

worldwide, almost all in low-income countries (LICs). ${ }^{1-3}$ Emergency obstetric and newborn care $(\mathrm{EmONC})$ is effective in treating obstetric complications and preventing maternal and perinatal mortality and morbidity. However, availability and quality of EmONC was found to be insufficient in most LICs. ${ }^{4}$

Ethiopia's maternal mortality ratio (MMR) was 412 per 100000 live births in 2016, a $39 \%$ reduction compared with an MMR of 676 in 2011. Its neonatal mortality rate showed a similar trend, with a reduction of $22 \%$ from 37 to 29 per 1000 live births. ${ }^{56}$ Although more women in Ethiopia gave birth at a facility than ever before, its institutional birth rate of $26 \%$ in 2016 (vs 10\% in 2011) still ranked among the lowest in the world. ${ }^{57}$ Many interventions are aimed at further improving women's access to maternity care, including expansion of the number of health facilities, distribution of ambulances and introduction of maternity waiting homes (MWHs). ${ }^{8}$ Pregnant women at low risk of complications are encouraged to stay at a MWH and deliver at a health centre, while those with risk factors should be referred to hospital. ${ }^{9}$ Even if risk selection is successful, 
Table 1 Signal functions of basic and comprehensive emergency obstetric care services

\begin{tabular}{lcc}
\hline EmONC signal functions & $\begin{array}{l}\text { Health } \\
\text { centres } \\
\text { (BEmONC) }\end{array}$ & $\begin{array}{l}\text { Hospitals } \\
\text { (CEmONC) }\end{array}$ \\
\hline $\begin{array}{l}\text { Administration of parenteral } \\
\text { antibiotics }\end{array}$ & $\checkmark$ & $\checkmark$ \\
$\begin{array}{l}\text { Administration of parenteral } \\
\text { uterotonics }\end{array}$ & $\checkmark$ & $\checkmark$ \\
$\begin{array}{l}\text { Administration of parenteral } \\
\text { anticonvulsants }\end{array}$ & $\checkmark$ & $\checkmark$ \\
\hline Manual removal of placenta & $\checkmark$ & $\checkmark$ \\
\hline Removal of retained products & $\checkmark$ & $\checkmark$ \\
\hline Assisted vaginal delivery & $\checkmark$ & $\checkmark$ \\
\hline $\begin{array}{l}\text { Neonatal resuscitation with } \\
\text { bag and mask } \\
\text { Blood transfusion }\end{array}$ & $\checkmark$ & $\checkmark$ \\
\hline Obstetric surgery & & $\checkmark$ \\
\hline
\end{tabular}

BEmONC, basic emergency obstetric and newborn care;

CEmONC, comprehensive emergency obstetric and newborn care; EmONC, emergency obstetric and newborn care.

$20 \%$ of low-risk pregnancies result in life-threatening complications requiring emergency treatment. ${ }^{10}$ Immediate access to basic emergency obstetric and newborn care (BEmONC) should, therefore, be guaranteed at health centres. In addition, a well-functioning referral system is essential to provide access to comprehensive care.

Previous EmONC assessments in Ethiopia have shown that only 1\%-2\% of health centres in 2008 and 2011 had provided all required life-saving services or 'signal functions' (see table 1). ${ }^{11}$ This study was undertaken at health centres in the Eastern Gurage Zone, Southern Ethiopia. In 2008, there were three functioning health centres in this zone, where in total 369 births took place. ${ }^{13}$

The objective of this study was to assess progress of BEmONC signal function provision, following major public investments to upgrade service delivery. We also aimed to assess knowledge of health workers regarding risk factors in pregnancy to assess feasibility of risk selection. Lastly, we aimed to determine the zone's referral capacity to establish if women in need of comprehensive care have appropriate access. Results from this study may help to further improve quality of BEmONC throughout the region. If Ethiopia and other countries in sub-Saharan Africa are successful in enhancing quality of BEmONC, women's usage of these services may increase, and maternal and neonatal mortality are likely to further decrease. ${ }^{14}$

\section{METHODS}

\section{Design and study area}

A cross-sectional survey was conducted at 20 health centres in the Eastern Gurage Zone, Ethiopia, in March and April 2015.

\section{Setting}

The study took place in the Eastern Gurage Zone, a predominantly rural and partly mountainous area in the Southern Nations and Nationalities and Peoples Region in Ethiopia. In Ethiopia, a zone is a mid-level administrative division of the country between region and district. The Eastern Gurage Zone is made up of four districts. During the study period, the zone had an estimated population of over 500000 of whom nearly 119000 were women in the reproductive age group (email from Sahle Kibru, Zonal Health Bureau Welkite (sahlekibru@yahoo.com) 2 May 2015 (cited 29 June 2017)). The national crude birth rate was 31.8 births per 1000 people; no regional data were available. ${ }^{5}$ Ethiopia's healthcare system is three-tier, comprising tertiary level (specialised hospitals), secondary level (general hospitals) and primary level (primary hospitals, health centres and health posts). At the time of the study, there were two hospitals (one government and one faith-based), 20 health centres and 119 health posts in the Eastern Gurage Zone. The hospitals had the option to refer to a tertiary hospital located in the capital, Addis Ababa, a three hour drive by ambulance.

The study took place in health centres. A health centre is a primary healthcare facility that serves a population of up to 25000 people. Staff working at a health centre include mid-level healthcare providers: health officers, midwives and nurses. The minimum staffing level at a health centre, set by the Ethiopian Standard Agency, is two health officers, three midwives and five nurses, alongside specialised supporting personnel (eg, laboratory and pharmacy technicians). ${ }^{15}$ In addition to routine preventive and curative maternal and neonatal health services, health centres are responsible for the provision of full BEmONC services. ${ }^{11}$ Additionally, the WHO has set a minimum standard of five EmONC facilities for a population of 500000 , including one that provides full comprehensive emergency obstetric and newborn care (CEmONC) services. ${ }^{16}$

\section{Study sites}

A total of 20 health centres were found in the Eastern Gurage Zone, which were all selected for the study. Health centres were included in the survey if they had provided delivery services in the 12 months prior to our study. One health centre was excluded as it had opened only one month prior to the site visit.

\section{Procedure}

A structured questionnaire was adapted from the Averting Maternal Death and Disability EmONC needs assessment tool, developed by Columbia University, New York and various partners of the United Nations. ${ }^{17}$ This extensively tested tool has proven successful in both countrywide and regional assessments. ${ }^{18-20}$ The purpose of the tool is to determine the existing capacity of health facilities to provide necessary life-saving care to pregnant women and neonates when complications occur. 
Our aim was to interview both the head of the health centre and the head of the maternity ward. In all but one health centre both were present during the interview; at one health centre we only interviewed the head of the maternity ward. Consequently, staff in all 19 health centres responded to our survey. Based on their performance in the 3 months prior to the survey, facilities were either classified as non-EmONC facility (no signal functions performed), 'partial-BEmONC' facility (one to six signal functions performed) or BEmONC facility (first seven signal functions performed) (table 1). In addition, modules were included on facility infrastructure and staffing. The availability of a labour ward, number of beds for obstetric patients, number of delivery beds and availability of a maternity waiting room or home were checked through observation. We recorded the number of medical doctors, health officers, midwives and nurses at each facility. Data on the number of attended deliveries, maternal deaths, intrauterine fetal deaths and early neonatal deaths in the 12 months prior to the survey were obtained using the health centres' monthly reports to the district health bureau, which are based on the health centres' labour ward registers. Furthermore, specific questions were formulated with regard to knowledge of pregnancy-related risk factors. Respondents were asked to spontaneously mention which women they would describe as being at risk of complications. In addition, they were asked which pregnant women they would admit to a MWH and which types of high-risk pregnancies they encounter most frequently. Respondents' answers were recorded and coded using a multiple-response set of 11 options (antepartum haemorrhage, hypertensive disorders, malpresentation, previous caesarean section, non-obstetric medical problems, breech presentation, distance from the facility, grand multiparity, anaemia, previous stillbirth and multiple pregnancy). Multiple choice questions were avoided since these may be vulnerable to guessing and do not test explicit knowledge. Lastly, a module was included on the facility's referral capacity. The questionnaire was originally drafted in English and then translated in Amharic, the national language of Ethiopia. It was then translated back into English to check for consistency. The questionnaire was pilot-tested on three employees of Butajira General Hospital.

The data collection team consisted of the first author and an experienced data collector from Butajira town. The data collector received additional training comprising the study objectives and methods, maternal health related topics, interviewing skills and ethical aspects of the study.

\section{Data analysis}

Completed questionnaires were checked for completeness and consistency on site. Data were entered into SPSS V.22 by the first author. Means and SD were calculated for continuous variables; frequencies and percentages for categorical variables. Simple linear regression was calculated to determine the relationship between the number of performed signal functions (dependent variable) and
Table 2 Infrastructure and staffing at health centres $(n=19)$

\begin{tabular}{ll}
\hline Available resources* & Health centres \\
\hline Infrastructure & \\
\hline Electricity & $13(68.4)$ \\
Running water & $12(63.2)$ \\
Labour ward & $19(100.0)$ \\
Postpartum room & $19(100.0)$ \\
Beds for obstetric patients & $2.3 \pm 2.1$ \\
Delivery couches & $2.7 \pm 1.1$ \\
MWH-available & $9(47.4)$ \\
MWH-under construction & $10(52.6)$ \\
Available skilled providers (mean) & \\
Health officers & $1.9 \pm 2.0$ \\
Midwives & $2.2 \pm 1.3$ \\
Nurses & $8.2 \pm 3.9$ \\
\hline
\end{tabular}

${ }^{*}$ Values are given as number (percentage) or mean \pm SD. $\mathrm{MWH}$, maternity waiting home.

the number of attended deliveries (independent variable), using a $95 \%$ CI.

\section{Consent to participate}

Written permission to conduct a health centre assessment in the Eastern Gurage Zone was given by the Zonal Health Bureau in Welkite, Ethiopia. Written informed consent was obtained from all respondents after explaining the purpose of the study, the importance of their contribution and the right to refuse participation. Questionnaires were stored anonymously to ensure respondents' confidentiality.

\section{RESULTS}

In total, 37 persons were interviewed. Eighteen were head of the health centre, of whom 17 were men. Out of the other 19 respondents, 14 were midwives, three were nurses, one was a health officer and one was designated as 'other'. Of these 19 respondents, 14 were women. The median age of the heads of the health centre was 27.5 years and they had a median 45 months of professional experience as a health worker. The other respondents had a median age of 24 years, while their median professional experience was 24 months.

\section{Infrastructure and staffing}

Table 2 summarises health centres' infrastructure and staffing. A total of 36 health officers, 41 midwives and 155 nurses were employed at the 19 health centres. Only three $(15.7 \%)$ health centres met the recommended staffing norm of two health officers, three midwives and five nurses. Ten thousand and four deliveries were attended to ( $\overline{\mathrm{X}}=527 \pm 301$ deliveries $)$, one to two deliveries per day per health centre on average. The number of deliveries attended ranged between 88 and 1086. Nineteen intrauterine fetal deaths and 13 early neonatal 


\begin{tabular}{lc}
$\begin{array}{l}\text { Table } 3 \\
\text { centres }(\mathrm{n}=19)\end{array}$ \\
$\begin{array}{lc}\text { Performed signal functions in the last } \\
\text { 3 months* }\end{array}$ & $\begin{array}{l}\text { Health } \\
\text { centres }\end{array}$ \\
\hline Parenteral administration of antibiotics & $8(42.1)$ \\
\hline Parenteral administration of uterotonics & $17(89.5)$ \\
Parenteral administration of anticonvulsants & $1(5.3)$ \\
Manual removal of placenta & $17(89.5)$ \\
Removal of retained products & $7(36.8)$ \\
Assisted vaginal delivery & $3(15.8)$ \\
Neonatal resuscitation with bag and mask & $17(89.5)$
\end{tabular}

*Values are given as number (percentage).

deaths were registered, corresponding to an institutional perinatal mortality rate of 3.2 per 1000 births, as well as eight maternal deaths, corresponding to an institutional MMR of 80 per 100000 live births.

\section{Performance of BEmONC signal functions}

No health centre had performed all seven basic signal functions in the 3 months prior to the survey, with a mean performance of $3.7 \pm 1.2$ signal functions. Results regarding provision of BEmONC signal functions are found in (table 3 and figure 1). In general, the most commonly cited reason for not performing a signal function was 'lack of a patient with an appropriate indication'. Health workers mentioned lack of training and supplies for not performing administration of parenteral anticonvulsants $(3 / 16.7 \%$ and $5 / 27.8 \%$, respectively) and for not performing assisted vaginal delivery $(6 / 37.5 \%$ and $8 / 50.0 \%$, respectively). The commonest reasons for not administering antibiotics were 'lack of a patient with an appropriate indication' $(10 / 90.1 \%)$ and lack of supplies $(3 / 27.3 \%)$. The number of performed signal functions did not correlate significantly with the number of attended deliveries $\left(\mathrm{F}^{1}{ }^{17}=2.297, \mathrm{P}<0.148\right.$, with an $\mathrm{R}^{2}$ of $0.119)$.

\section{Knowledge of risk factors for complications}

On average, $3.9 \pm 1.4$ risk factors for complications during labour or delivery were mentioned by respondents (figure 2). Two of the three risk factors mentioned most frequently were also said to be the most commonly

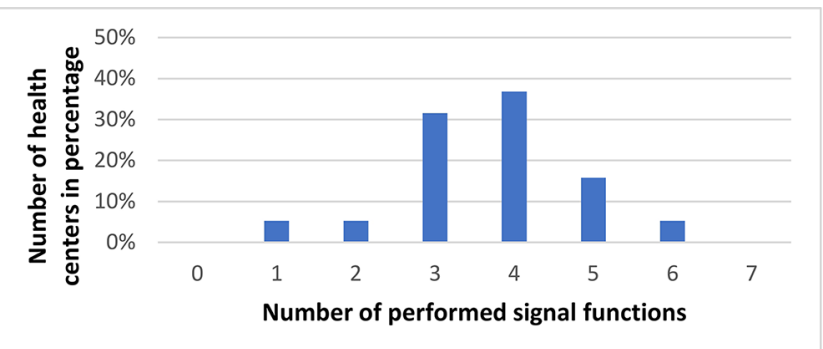

Figure 1 Number of BEmONC* signal functions performed at health centres $(n=19)$; ${ }^{*} \mathrm{BEmONC}$ - basic emergency obstetric and newborn care. encountered: hypertensive disorders $(8 / 42.1 \%)$ and antepartum haemorrhage $(6 / 31.6 \%)$. Distance from health facility $(16 / 84.2 \%)$ and hypertensive disorders $(13 / 68.4 \%)$ were stated most often as indications for MWH admission. At eight health centres $(42.1 \%)$, at least one health worker $(\overline{\mathrm{X}}=2.0 \pm 1.1)$ had received training on how to diagnose high-risk pregnancies. Of the 41 midwives, $11(26.8 \%)$ had received training for this purpose.

\section{Referral capacity}

Eighteen $(94.7 \%)$ health centres had referred highrisk pregnant and labouring women to a higher-level facility. For referral purposes, staff used their own mobile phones, as only five $(26.3 \%)$ had a working landline telephone and one $(5.3 \%)$ facility owned a mobile phone. In total, five ambulances were available for the Eastern Gurage Zone population. Two ambulances were stationed at health centres, three were stationed at District Health Offices and shared among the other 17 health centres. When ambulances were not available, nine (47.4\%) health centres indicated using public transport to transfer labouring women. The majority of health centres $(10 / 52.6 \%)$ were difficult to access as these were located in mountains or arid lowlands, accessible by unpaved roads only. The median distance to Butajira General Hospital, the referral hospital used by all health centres, was $16.5 \mathrm{~km}$.

\section{DISCUSSION}

In response to a very low proportion of facility births, the main strategy of the Ethiopian Ministry of Health has been to scale up the number of health centres, as illustrated by a more than sixfold increase in the number of such facilities in just seven years in this specific zone. Our study provides insight into whether these facilities (are able to) provide life-saving BEmONC services. Moreover, our study is the first facility-based BEmONC assessment in rural Ethiopia since the introduction of MWHs aimed at enhancing uptake of facility-based birth care. Our results indicate that, although most health centres provide delivery care on a daily basis, none could be classified as fully functional BEmONC facilities. In addition, lack of knowledge about pregnancy-related risk factors among health workers and a resource-constrained referral system prevent women from arriving at the appropriate level of care in case they require CEmONC.

Compared to the 2008 national EmONC assessment, the number of health centres in the Eastern Gurage Zone increased from 3 to 20 and the number of ambulances from one per eight health centres to one per four. ${ }^{13}$ The number of professional health staff also increased, from 31 to 234 . However, the average staffing level per health centre remained the same and most health centres did not meet the 2012 staffing norms. ${ }^{15}$ Women are increasingly seeking facility-based birth care, since the average annual number of attended births per health centre has 


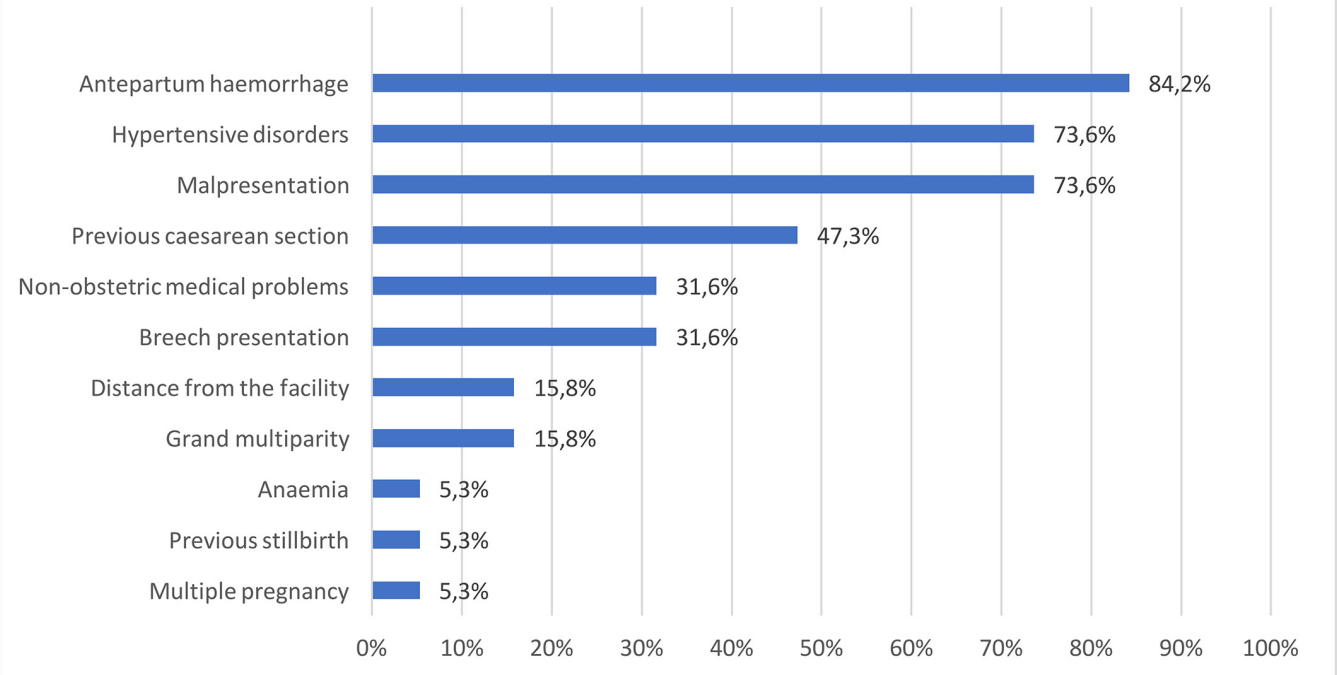

Figure 2 Knowledge at health centres regarding risk factors for high-risk pregnancies $(n=19)$.

increased by five times. The three health centres that were functional in 2008 did not improve with regard to the number of performed signal functions (four to five). Overall performance in the zone was low, with an average of 3.7 signal functions performed. Poorest performance was found on administration of parenteral anticonvulsants and assisted vaginal delivery. These findings are largely in line with earlier EmONC assessments in Ethiopia, the main difference being that our surveyed health centres performed better on neonatal resuscitation. ${ }^{11} 1221$ Our findings also correlate with EmONC assessments in other countries. Between 2009 and 2011, 218 health facilities that were expected to provide BEmONC services were surveyed in six countries with medium to high maternal mortality. Only five $(2.3 \%)$ of the health facilities provided all BEmONC services. In three countries, none of the surveyed health facilities provided full BEmONC. ${ }^{22}$ In this study, the most frequently mentioned reason for nonperformance was lack of patients with the appropriate indication. However, no relationship was found between the number of attended deliveries and the number of signal functions performed. Underperformance, therefore, likely also reflects lack of knowledge and competence (or confidence) to recognise and treat complications. Given the recent expansion of health facilities and health workers in Ethiopia, focus on quality of care should now be urgently intensified. This is especially important since women are encouraged to stay at $\mathrm{MWH}$ at health centre level. If women are given the option to stay at a MWH, immediate access to emergency care must be guaranteed, both from a medical and an ethical perspective. ${ }^{10}$

Health centre staff play a crucial role in risk selection, to ensure that women give birth at the right level of care. Health workers are expected to select low-risk pregnant women for a 2-week stay at their MWH to await birth in close proximity to skilled care, and refer high-risk pregnant women to stay at a MWH adjacent to hospital. Respondents in our survey scored worse on knowledge of high-risk pregnancies than those in the 2008 assessment (3.9 risk factors out of 11 options compared with 3.4 risk factors out of 8 options). While the two surveys used different options and only three options were exactly the same, our results show that improving knowledge among staff of risk during pregnancy and labour is a key priority. ${ }^{13}$ Even if risk selection is successful and full BEmONC services are provided, referral options are needed when women need comprehensive care. Since 2012, the Ethiopian government has distributed one ambulance per district and two for larger districts. Usage of these ambulances has reduced pregnancy-related deaths. ${ }^{23}{ }^{24}$ Our study area met the target number of one ambulance per district. We recommend further expanding the number of ambulances in areas that are difficult to access, such as the Eastern Gurage Zone.

Based on the national Crude Birth Ratio of 31.8 per 1000 people and an estimated population of 500000 , an expected 15900 deliveries occurred in 1 year in the Eastern Gurage Zone. This study reported 10004 health centre births. Between 2011 and 2014, the average annual number of deliveries at Butajira Hospital was 2368 (Braat, unpublished data, 2017). This would correspond to an institutional delivery rate in the zone of at least $78 \%$, which is substantially higher than the reported $26 \%$ in the EDHS for 2016. Further research into this discrepancy is needed.

WHO has indicated that one of the greatest challenges LICs face are implementation challenges. ${ }^{25}$ We evaluated BEmONC provision, knowledge of high-risk pregnancies and referral options at all health centres in one area in Ethiopia. By including multiple aspects of delivery care services, stakeholders can take targeted action to improve quality of care on different levels within the Eastern Gurage Zone.

A small-scale assessment such as this study is a timeefficient and resource-efficient means to monitoring progress throughout the wider region. It is unlikely that 
in similar facilities in other areas results would be much different. However, our findings should be interpreted with caution because interviews with health centre staff are vulnerable to recall bias, and the assessed health centres are limited in number and to one zone only. It should also be noted that while we make comparisons between our study and the 2008 EmONC assessment, the region we studied had only three functioning health centres in 2008 compared with 20 health centres in 2015. The comparisons are made between the 20 health centres we surveyed and a total of 650 health centres surveyed in 2008. This should be considered a limitation. However, the findings in our study are consistent with other assessments done in Ethiopia and other LICs. We, therefore, think that our findings can be extrapolated to other LICs with high maternal mortality rates. Reassessments should capture performance on the newly proposed signal functions by Gabrysch $e t$ al, which were introduced following the growing consensus that more focus is needed on routine care to prevent complications. These include the use of the partograph, active management of third stage of labour and infection prevention measures. ${ }^{26} 27$ Checking (non-)availability of supplies, drugs and equipment would also provide a more comprehensive understanding of EmONC performance and the challenges involved in providing quality care. Reassessments should also include a more thorough examination of the referral system. This study did not capture information about the efficient use of ambulances and other resources, prereferral treatment or timeliness of referrals.

\section{CONCLUSION}

Conducting regular systematic assessments of EmONC at health facilities is important in order to evaluate the availability of essential life-saving services to mothers and newborns. While most health centres in our study area provided delivery services on a daily basis, none provided all BEmONC services. This study shows that there is an urgent need to train health centre staff in the identification of high-risk pregnancies and BEmONC, specifically on diagnosing and treating (pre-)eclampsia and performance of assisted vaginal delivery. Women who are encouraged to give birth in a facility should be able to give birth safely, attended by health centre staff knowledgeable and skilled to recognise high-risk pregnancies and to provide BEmONC, and have access to emergency transport if more comprehensive care is needed.

Acknowledgements We wish to thank employees of the health centres and Butajira hospital for their cooperation in this study.

Contributors MW, TV, FB, AMT, AG and JS conceived and designed the study. MW led data collection. Data analysis was done by MW and TV. FB, TA and JS helped interpret the data. MW and TV drafted a first manuscript, which was then edited by all other authors. All authors read and approved the final manuscript.

Funding Laerdal Foundation (grant number 40171) supported this study as part of a PhD project of investigator TV. Investigator FB received funding from VSO Ethiopia and Otto Kranendonk Foundation for data collection and Open Access publication.

Competing interests None declared.
Ethics approval Southern Nations Nationalities and People Regional State Health Bureau in Hawassa, Ethiopia on 4 February 2014, with reference number 1-1/9466.

Provenance and peer review Not commissioned; externally peer reviewed.

Data sharing statement The data in support of the conclusions of this paper are included in the paper. Further information on the survey and dataset can be requested from the authors.

Open Access This is an Open Access article distributed in accordance with the Creative Commons Attribution Non Commercial (CC BY-NC 4.0) license, which permits others to distribute, remix, adapt, build upon this work non-commercially, and license their derivative works on different terms, provided the original work is properly cited and the use is non-commercial. See: http://creativecommons.org/ licenses/by-nc/4.0/

(C) Article author(s) (or their employer(s) unless otherwise stated in the text of the article) 2017. All rights reserved. No commercial use is permitted unless otherwise expressly granted.

\section{REFERENCES}

1. World Health statistics 2016: Child Mortality. Monitoring health for the SDGs. World Health Organization. 2016.

2. World Health Organization. Maternal, newborn, child and adolescent health. http://www.who.int/maternal_child_adolescent/epidemiology/ stillbirth/en/ (acccessed 14 Apr 2017).

3. World Health Organization. Trends in maternal mortality: 1990 to 2015: estimates by WHO, UNICEF, UNFPA, World Bank Group and the United Nations Population Division. Geneva: World Health Organization, 2015.

4. Campbell OM, Calvert C, Testa A, et al. The scale, scope, coverage, and capability of childbirth care. Lancet 2016;388:2193-208.

5. Central Statistical Agency Ethiopia. Ethiopia demographic and health survey 2016. Addis Ababa, Ethiopia. 2016.

6. Central Statistical Agency Ethiopia. Ethiopia demographic and health survey 2011. Addis Ababa, Ethiopia. 2011.

7. Births attended by skilled health staff. Internet site World Bank. http://data.worldbank.org/indicator/SH.STA.BRTC.ZS (accessed 12 Apr 2017).

8. Overview of the Ministry. Internet site ministry of health, Government of Ethiopia. http://www.moh.gov.et/overviewministry (accessed 20 Apr 2017).

9. Federal Ministry of Health Ethiopia. Guideline for the establishment of standardized maternity waiting homes at health centers/ facilities. Addis Ababa, Ethiopia. 2015.

10. van Lonkhuijzen L, Stekelenburg J, van Roosmalen J. Maternity waiting facilities for improving maternal and neonatal outcome in low-resource countries. Cochrane Database Syst Rev 2012;10:CD006759.

11. Admasu K, Haile-Mariam A, Bailey P. Indicators for availability, utilization, and quality of emergency obstetric care in Ethiopia, 2008. Int J Gynaecol Obstet 2011;115:101-5.

12. JSI Research and Training Institute: The Last 10 Kilometers (L1OK). Emergency referral for pregnant women and newborns: A rapid community and health system assessment. Addis Ababa, Ethiopia, 2012.

13. Federal Ministry of Health Ethiopia. National baseline assessment for emergency obstetric \& newborn Care 2008. National report and Woreda fact sheet of the gurage zone. Ethiopia: Federal ministry of health, UNICEF, UNFPA, WHO, AMDD. 2010.

14. Jonas K, Crutzen R, van den Borne B, et al. Healthcare workers' behaviors and personal determinants associated with providing adequate sexual and reproductive healthcare services in subSaharan Africa: a systematic review. BMC Pregnancy Childbirth 2017;17:86.

15. Ethiopian standard agency. Ethiopian standards, health centre requirements, 2012. ES:3611.

16. WHO,UNFPA, UNICEF \& AMDD. Monitoring emergency obstetric care: a handbook, 2009.

17. Averting Maternal Death and Disability (AMDD). Needs assessment of emergency obstetric and newborn care: data collection modules. New York: Columbia University, UNFPA, UNICEF, and WHO. 2010.

18. Fakih B, Nofly AAS, Ali AO, et al. The status of maternal and newborn health care services in Zanzibar. BMC Pregnancy Childbirth 2016;16:134.

19. Keyes EB, Haile-Mariam A, Belayneh NT, et al. Ethiopia's assessment of emergency obstetric and newborn care: setting the gold standard for national facility-based assessments. Int J Gynaecol Obstet 2011;115:94-100. 
20. Solnes Miltenburg A, Kiritta RF, Bishanga TB, et al. Assessing emergency obstetric and newborn care: can performance indicators capture health system weaknesses? BMC Pregnancy Childbirth 2017;17:92.

21. Worku AG, Yalew AW, Afework MF. Availability and components of maternity services according to providers and users perspectives in North Gondar, Northwest Ethiopia. Reprod Health 2013;10:43.

22. Ameh C, Msuya S, Hofman J, et al. Status of emergency obstetric care in six developing countries five years before the MDG targets for maternal and newborn health. PLoS One 2012;7:e249938.

23. Godefay H, Kinsman J, Admasu K, et al. Can innovative ambulance transport avert pregnancy-related deaths? One-year operational assessment in Ethiopia. J Glob Health 2016;6:010410.
24. Tsegaye A, Somigliana E, Alemayehu T, et al. Ambulance referral for emergency obstetric care in remote settings. Int J Gynaecol Obstet 2016;133:316-9.

25. Peters DH, Tran N, Adam T, et al. Implementation research in health: a practical guide. Alliance for Health Policy and Systems Research, World Health Organization, 2013.

26. Banke-Thomas A, Wright K, Sonoiki O, et al. Assessing emergency obstetric care provision in low- and middle-income countries: a systematic review of the application of global guidelines. Glob Health Action 2016:9:31880.

27. Gabrysch S, Civitelli G, Edmond KM, et al. New signal functions to measure the ability of health facilities to provide routine and emergency newborn care. PLoS Med 2012;9:e1001340. 